\title{
Growth of the Tooth Germ in Newborn Rats Exposed to Oxygen at Atmospheric and High Pressure
}

\author{
T. NAKAMOTO* and J. W. BEAN
}

Department of Physiology, Medical School and Department of Oral Biology, School of Dentistry, The University of Michigan, Ann Arbor, Michigan 48104, USA

Newborn rats were exposed intermittently to oxygen at high pressure $(93$ psig) 15 times for a period of eight days. Growth of the tooth germs was the same as that of the control rats in air at atmospheric pressure, with the exception of the enamel and dentin layers, which grew less. The width of the tooth germ of rats exposed to oxygen at atmospheric pressure increased.

The exposure of adult rats to oxygen at high pressure precipitates convulsive seizures. ${ }^{1,2}$ It seems that such exposures have an increased growth effect on the incisor ${ }^{3}$ and on the tooth germ of rat embryos in the uterus of mothers subjected to such seizures. ${ }^{4}$ In contrast, intermittent exposures to oxygen at atmospheric pressure promotes increased body weight of adult rats and embryos, ${ }^{3-5}$ but does not modify the growth rate of either the incisors or the tooth germ. ${ }^{3,4}$ We have suggested ${ }^{3,4}$ that this increased growth rate of the incisors and of the tooth germ may have been caused by the adrenal glands. It is recognized generally that adrenal glands have a protective influence in stress conditions; however, in contrast to this, adrenalectomy affords some protection against the stress of oxygen at high pressure. ${ }^{6}$ Newborn rats (in which the adrenal glands are not fully developed) were used to test the hypothesis that alteration of tooth germ growth by oxygen at high pressure is caused by the adrenal glands. Experiments were carried out to determine whether oxygen at atmospheric pressure or oxygen at high pressure modifies the growth

\footnotetext{
This investigation was supported in part by a Grant H.E. 01646 and USPHS DE-00013 from the National Institutes of Health, Bethesda, Md.

Received for publication April 27, 1972.

* Present address: Massachusetts Institute of Technology, Cambridge, Mass.
}

of the tooth germ, modifies the cell layers of the tooth germ, including enamel and dentin, or affects body weight.

\section{Materials and Methods}

Three experimental environments were used: air at atmospheric pressure (AAP), oxygen at atmospheric pressure (OAP), and oxygen at high pressure (OHP). The chambers for the OAP and OHP exposures were described previously. ${ }^{4}$ Soda lime was spread on the floor of the chambers to prevent carbon dioxide accumulation. The levels of carbon dioxide and oxygen were analyzed carefully with the instruments described previously. ${ }^{4}$ The level of the carbon dioxide was maintained at less than $0.1 \%$. The temperature of the chambers throughout the experiment was about $27 \mathrm{C}$.

Fifty-two Sprague-Dawley newborn rats from six mother rats were used. Within 12 hours after delivery, they were separated at random into three groups: 16 for AAP, 18 for OAP, and 18 for OHP. Two mother rats were assigned to each group. At the beginning of the experiment the chambers were washed free of air by the rapid flow of oxygen.

The newborn rats in the first group were exposed intermittently to OHP an average of twice daily for a total of 15 times. The pressure range was from 80 to 110 pounds per square inch gauge (psig), with an average of about 93 psig ( 7.2 atmospheres absolute). The usual range of compression time was about three minutes and the duration of the exposures was about 12 minutes. Decompression was carried out in stages over a period of 19 minutes. The group exposed to OAP followed an identical program to that of the group exposed to OHP, that is, about 34 minutes exposure 
15 times over a period of seven days. Newborn rats of the group exposed to AAP were separated from the mothers during the OAP and OHP exposures, and placed in a separate cage to ensure the same nursing time for all three groups. The rats were weighed every other day for a period of eight days. Newborn rats of extremely low weight, possibly caused by nursing failure, were discarded.

The mothers were given a standard laboratory diet and water ad libitum throughout the experimental period. On the eighth day, ten newborn rats were selected randomly from each group and given an overdose of sodium pentobarbital. The right and left sides of the mandibles were removed under the dissection microscope, fixed with Bouin's solution, and embedded in paraffin. Sagittal sections 10 micrometers $(\mu \mathrm{m})$ thick were made of the mandibular structures from the buccal to the lingual aspect. Every tenth section was placed on a glass slide and stained with hematoxylin and eosin. The size of the tooth germ, ameloblast, enamel, dentin, and odontoblast layers were measured by the method described previously.$^{4,7}$ The tooth germ and the body of the mandible were examined microscopically to determine if any changes occurred.

The data were analyzed by use of analysis of variance ( $F$ test) and Scheffe's test. A difference at the $5 \%$ level was considered statistically significant.

\section{Results}

The data obtained from male and female newborn rats were pooled because statistical analysis showed no significant difference attributable to sex $(P>0.05)$. Three of the newborn rats exposed to OAP died. On the second day, the rats exposed intermittently to OHP weighed less than those of the control group (AAP) $(P<0.01)$. However, on the subsequent days, that is, the fourth, sixth, and eighth days, the increase of the average weight in the OHP group was the same as that of the controls (AAP) $(P>$ 0.05 ) (Fig 1). The width and height of the tooth germ in the OHP group were the same as those of the controls (AAP) $(P>0.05)$ (Fig 2). The thickness of the ameloblast layer in the OHP group was not statistically different from that of the controls (AAP) $(P>0.05)$ (Fig 3); however, the thickness of the enamel and dentin layers in the OHP group was less $(P<0.01)$ (Fig 3$)$. The thickness of the odontoblast layer in the OHP group was greater than that of the controls (AAP) $(P<0.01)$ (Fig 3).

The body weight of newborn rats that were exposed intermittently to OAP in-

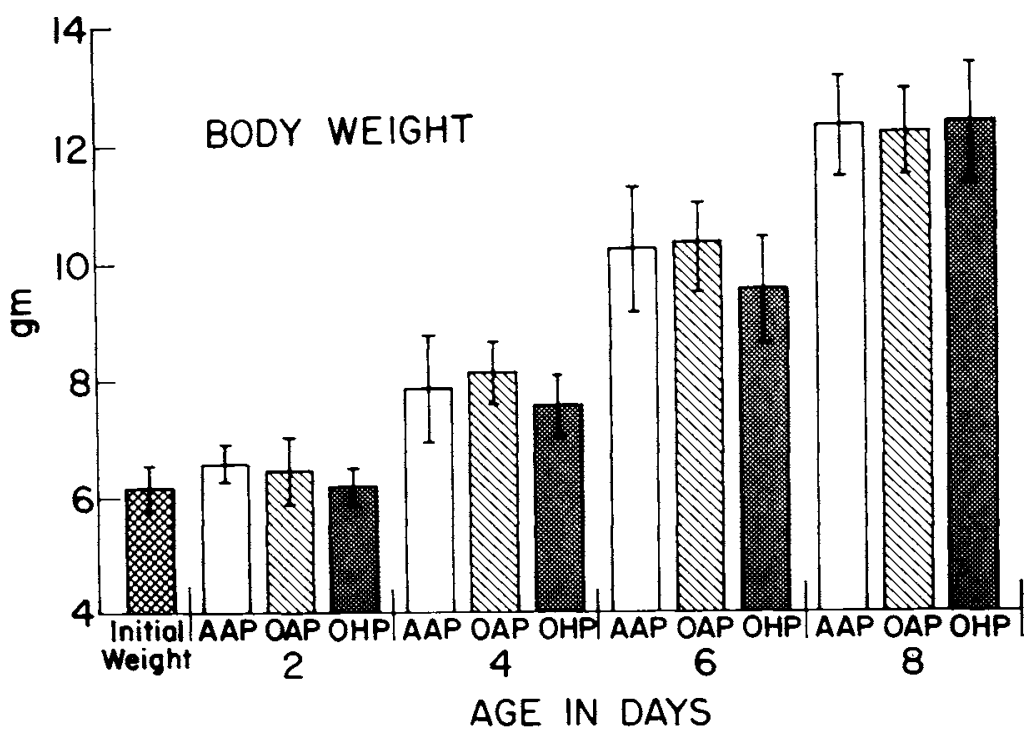

Fig 1.-Average body weight of newborn rats during first eight days after birth. 

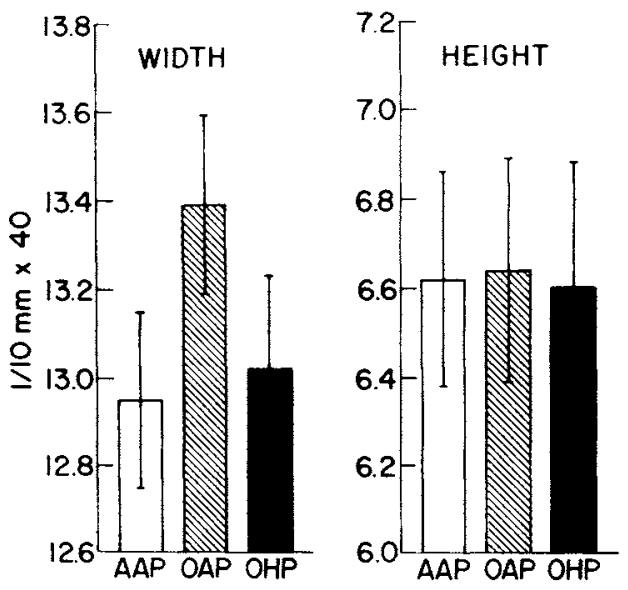

FIg 2.-Comparison of average width and height of tooth germ of first molar of newborn rats exposed to AAP, OAP, and OHP. Numerals on ordinate represent measurement from photographic enlargement factor. Actual size of 12.6 equals $0.315 \mathrm{~mm}$. Each bar represents average of 55 to 65 measurements.

creased at the same rate as that of the controls throughout eight days of the experimental period $(P>0.01)$ (Fig 1). The width of the tooth germ in the OAP group was greater than that of the controls (AAP) $(P<0.01$ ) (Fig 2), but the height of the tooth germ was not statistically different $(P>0.05) \quad($ Fig 2$)$. The thickness of the ameloblast, enamel, and dentin layers in the OAP group was the same as that of the controls (AAP) $(P>0.05)$ (Fig 3), but the thickness of the odontoblast layer was greater $(P<0.05)$ (Fig 3$)$. Sections of the tooth germ and the body of the mandible did not show any pathologic signs such as hyperemia, decompressional bubble formation, or hemorrhage.

\section{Discussion}

Data show that the body weight of newborn rats exposed intermittently to OHP for a period of eight days is about the same as that of control rats. In contrast to this, the body weight of the adult rats subjected to similar OHP exposures decreased..$^{3}$ This suggests that newborn rats are more resistant to the adverse effects of OHP than adult rats.

It has been shown that the hypophysis and the adrenal glands play an important role in oxygen toxicity of rats exposed to OHP. ${ }^{6,8}$ For example, adrenalectomy or hypophysectomy provide some protection, such as delayed onset of convulsive seizure, decreased severity of the acute neuromuscular response, and less pulmonary damage. ${ }^{6,8}$ This greater resistance of newborn rats may be caused by the underdevelopment of the hypophysis and adrenal glands. It is known that the growth hormone released from the hypophysis has an indispensable role in the later growth of rats, but it may not be essential to early postnatal growth. ${ }^{9-11}$ It also has been shown that the adrenal glands of
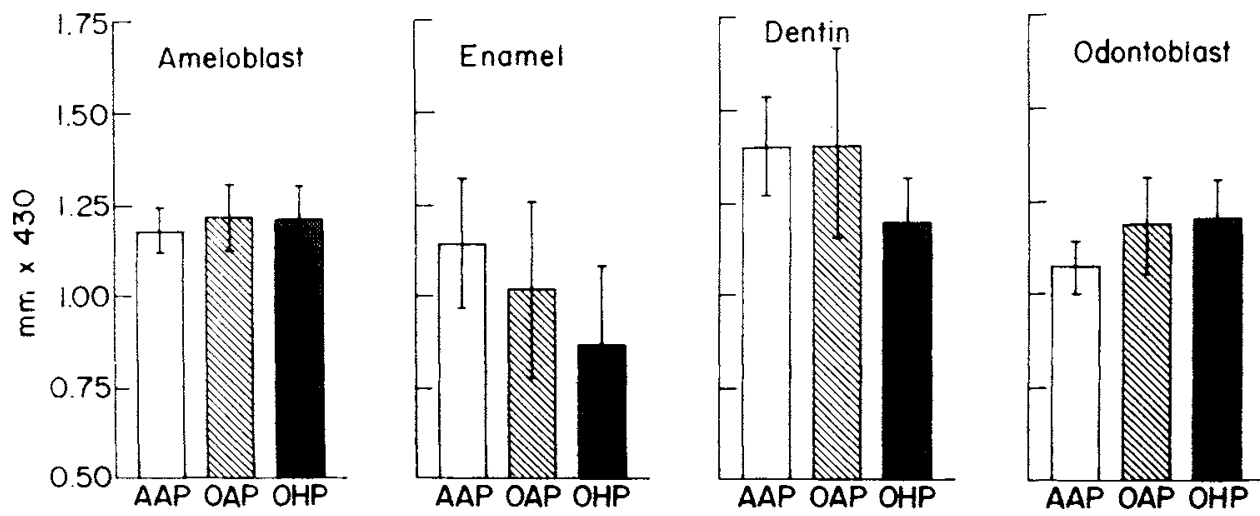

FIG 3.-Comparison of thickness of ameloblast, enamel, dentin, and odontoblast layers of tooth germ of newborn rats exposed to AAP, OAP, and OHP. Measurements were made using an ocular microscope at magnification of 430 . Number of scales is arbitrary, actual size of 10 equals $0.5 \mathrm{~mm}$ under 430 magnification. Each bar represents average of 55 to 65 measurements. 
newborn rats are not fully developed until the 16th day after birth. ${ }^{12}$ Previous studies have shown that the tooth growth in young adult rats subjected to oxygen convulsions was greater than that of the controls, ${ }^{3}$ and also that tooth germs of embryos in the uterus while the mothers were subjected to oxygen convulsion increased similarly. ${ }^{4}$ But in this experiment with newborn rats (up to 8 days old) that had no convulsions, tooth germ growth was not greater than that of the controls.

It seems, therefore, that tooth growth in the young adult rats was altered by oxygen convulsions, possibly through the release of some unknown factor. Oxygen convulsions in mothers also altered the tooth germ growth of embryos in the uterus. Since there was no change in tooth germ growth in the absence of convulsions, it might be expected that the ameloblast and odontoblast layers, as well as enamel and dentin formation, also would be unchanged. However, the enamel and dentin layers in the OHP group were thinner than those of the controls (AAP), and the odontoblast layer was thicker; there was no change in the ameloblast layer.

The body weight of newborn rats exposed intermittently to OAP for a period of eight days was the same as that of the control rats (AAP) in these experiments. Continuous exposures to OAP in newborn rats may retard the growth of the tooth germ. ${ }^{13}$ However, the width of the tooth germ of newborn rats exposed intermittently to OAP was greater than that of the controls (AAP), although its height was not altered. The cause of this pronounced difference between growth in width and height is not known. The thicknesses of the ameloblast layer, enamel, and dentin in the OAP groups were not statistically different than those of the controls $(P>0.05)$, but the thickness of the odontoblast layer increased. It seems that OAP and OHP exert a specific influence on the ameloblast and odontoblast cells, which might affect enamel and dentin formation. The difference between the results of these and earlier experiments ${ }^{3,4}$ involving OAP and OHP exposures may be caused by the unknown influence of the hypophysial adrenal axis in newborn rats. Histologic examination of the tooth germ in the present experiment revealed no pathologic change such as hyperemia or hemorrhage.

\section{Conclusions}

The body weight, width, and height of the tooth germ and the thickness of the ameloblast layer of newborn rats exposed to oxygen at high pressure (93 psig) for 34 minutes twice daily for the first seven days after birth were not significantly different than those of control rats (AAP). Enamel and dentin formation in these rats were less than that of the controls, but the thickness of the odontoblast layer increased. In parallel exposures to oxygen at atmospheric pressure, the body weight, height of the tooth germ, thickness of the ameloblast, enamel, and dentin layers were the same as that of the controls, but width of the tooth germ and odontoblast layers increased. We concluded that influence of oxygen tension is not uniform in all phases of the development of the tooth germ in newborn rats. This may be related to the undeveloped hypophysial adrenal axis at this age.

\section{References}

1. BEAN, J.W.: Effects of Oxygen at Increased Pressure, Physiol Rev 25:1-147, 1945.

2. Thomson, W.A.R.: The Physiology of Deep Sea Diving, Br Med J 2:208-210, 1935.

3. NaKamoto, T., and BEAN, J.W.: Differences in Growth Pattern of Bone and Incisor of Rats Exposed to $\mathrm{O}_{2}$ at Atmospheric and High Pressure, Arch Oral Biol 17: 799, 1972.

4. Nakamoto, T., and Bean, J.W.: Influence of Oxygen at Atmospheric and High Pressure on the Developing Tooth Germ in Rat Embryos, J Dent Res 52:1397-1403, 1972.

5. Dines, J.H., and HiatT, E.P.: Prolonged Exposure of Young Rats to an Oxygen Atmosphere at Reduced Pressure, J Appl Physiol 19:17-20, 1964.

6. BEAN, J.W., and JoHNSON, P.C.: Adrenocortical Response to Single and Repeated Exposure to Oxygen at High Pressure, Am J Physiol 179:410-414, 1954.

7. Nakamoto, T., and Wilson, R.H.: The Growth Rate of Tooth Germ in NewlyBorn Rats Injected with Cortisone Acetate, Arch Oral Biol 13:1107-1118, 1968. 
8. BEAN, J.W.: The Hypophysis as a Determinant in the Reaction of the Mammal to $\mathrm{O}_{2}$ at High Pressure, Am J Physiol 170:508-517, 1952 .

9. Evans, H.M.: The Hormone of the Anterior Pituitary, Am J Orthod 32:472-478, 1946.

10. Nakamoto, T, and Wilson, R.H.: The Effect of Wave Length and Light Intensity on the Growth of Tooth Germ in Newborn Rats, Arch Oral Biol 15:195-204, 1970.

11. VAN ECK, W.F., and FREUD, J.: Analysis of Growth of Very Young Hypophysec- tomized Immature Rats, Acta Brev Neerl 11:43-46, 1941.

12. BARRY, L.W.: The Effects of Initiation in the Pregnant Albino Rat, with Special Reference to the Changes in the Relative Weights of the Various Parts, Systems, and Organs of the Offsprings, in Contributions to Embryology Vol 11, No. 53, Washington, DC: The Carnegie Institution of Washington, 1920, $122 \mathrm{pp}$.

13. Fitzgerald, L.R.; Cohn, S.A.; Page, C.; and SrCkIER, J.: L'action de l'oxygéne sur la formation dentaire, Med Hyg (Geneva) 26:143, 1968. 